\title{
DIE DEUTSCHE SPRACHE IN FRANKREICH, MIT BESONDERER BERÜCKSICHTIGUNG DES ELSASS - EIN HISTORISCHES THEMA UND SEINE AKTUELLEN ABLEITUNGEN
}

\section{PAUL LÉVY (1887-1962) UND „DIE DEUTSCHE SPRACHE IN FRANKREICH“"}

In den Jahren 1950 und 1952 erschien die zweibändige Geschichte der deutschen Sprache in Frankreich aus der Feder des Germanisten und Historikers Paul Lévy (Lévy 1950/52). ${ }^{1}$ Aus einer jüdisch-elsässischen Familie stammend, war Lévy von seinem Studium her gleichermaßen von der deutschen wie von der französischen Germanistik der Zeit vor dem Ersten Weltkrieg geprägt. Er schrieb sich 1906 im damals zu Deutschland gehörenden Elsass an der Universität Straßburg ein, an der er zum Abschluss seines Studiums 1910 auch auf Grundlage einer germanistischen Dissertation über den Begriff des Volksliedes und seine Geschichte promoviert wurde (Lévy 1911). Das Studienjahr 1907/08 verbrachte er jedoch in Paris, wo er unter anderem an den Lehrveranstaltungen des bedeutenden Germanisten Charles Andler (1866-1933) teilnahm. Andler stammte aus einer wohlhabenden Straßburger Familie, war aber als Jugendlicher zu seiner weiteren Ausbildung nach Frankreich gegangen und hatte hier die Qualifikationen für den Schul- und den Hochschuldienst erworben. Andler, der sich in diesen Jahren sehr für die Philosophie Friedrich Nietzsches interessierte, veranlasste seinen Studenten dazu, einige NietzscheBriefe ins Französische zu übersetzen (Lévy 1908). Noch größeren Einfluss übte auf Lévy zweifellos der hervorragende Sprachwissenschaftler Ferdinand Brunot (1860-1933) aus, für den 1899 an der Sorbonne eigens ein Lehrstuhl für französische Sprachgeschichte eingerichtet worden war. Brunots monumentale „Geschichte der französischen Sprache“ erschien ab 1905 und wuchs im Lauf der Jahre zu einem Maßstäbe setzenden Standardwerk heran (Hafner 2006). ${ }^{2}$ Brunots Methode, die darin bestand, die innere Sprachgeschichte (histoire interne) stets auf die äußere Sprachgeschichte (histoire externe) zu beziehen und in starkem Maße kulturelle und gesellschaftliche Entwicklungen zu berücksichtigen

thomas.nicklas@univ-reims.fr

1 Der erste Band des Werkes behandelte die Geschichte der deutschen Sprache in Frankeich bis zum Jahr 1830, während der zweite Band bis in die Zeit des Zweiten Weltkrieges reichte. Inzwischen liegt auch eine vorzügliche deutsche Übersetzung der beiden Bände vor: Kaltz 2013/2016. Dort auch, Bd. 1 (Kaltz 2013), XIX-XXIV, weitergehende Ausführungen zur Rezeptionsgeschichte des Werkes.

2 Maßgeblich ist die 1933 erschienene vierte Auflage des Werkes, von der ein Nachdruck vorliegt: Brunot (1933). 
(Hafner 2006: 156-158), hat die künftigen Arbeiten Paul Lévys zur Geschichte des Deutschen in Frankreich stark beeinflusst.

Seit 1911 war Lévy im Schuldienst des Reichslandes Elsass-Lothringen tätig. Ab 1914 wirkte er als Gymnasiallehrer im Fach Deutsch im lothringischen Diedenhofen und setzte diese Laufbahn an derselben Schule nach 1919 im nun wieder französischen Thionville fort. Schließlich konnte er an das Lycée Kléber in Straßburg wechseln, wo er bis zum Jahre 1933 verblieb. Neben der Schultätigkeit widmete sich Paul Lévy weiterhin seinen sprachgeschichtlichen Forschungen und publizierte unter anderem über die in Lothringen gesprochenen germanischen Dialekte (Lévy 1923). An der Universität Straßburg erfolgte 1929, nicht ohne gewisse Schwierigkeiten, die Habilitation des ganz wesentlich vom deutschen Hochschulwesen geprägten Germanisten und Historikers Paul Lévy (Kaltz 2013: XIII-XVI), für die er eine umfassende Darstellung der Sprachgeschichte von Elsass und Lothringen in zwei Bänden vorgelegt hatte (Lévy 1929). Allerdings sollten sich seine Hoffnungen auf eine Hochschullaufbahn nicht erfüllen. Stattdessen beantragte und erhielt Lévy 1933 eine Versetzung an ein angesehenes Gymnasium in Paris (Lycée Rollin). Seine Forschungen galten daneben weiterhin der deutschen Sprache und ihrer Wahrnehmung in Frankreich, beispielsweise bei den Dichtern der französischen Romantik (Lévy 1938).

Nach der deutschen Besetzung Frankreichs verlor Paul Lévy im Herbst 1940 aufgrund der antijüdischen Gesetzgebung seine Stelle im Schuldienst. Schließlich musste die Familie Lévy in den Untergrund gehen und überlebte die Besatzungszeit mit Mühe und Not in wechselnden Verstecken. Nach der Befreiung der französischen Hauptstadt konnte er jedoch bereits im September 1944 die Lehrtätigkeit an seiner Schule wieder aufnehmen. Aufgrund seiner Verfolgung in der Zeit des Nationalsozialismus war Lévy nach 1945 berechtigt, um in glaubwürdiger Weise für den Deutschunterricht in Frankreich und für eine deutsch-französische Annäherung einzutreten (Kaltz 2013: XVIII). In späteren Jahren galt sein wissenschaftliches Interesse nicht zuletzt der Sprache und der Kultur der französischen Juden. Zwei Jahre vor seinem 1962 erfolgten Tod publizierte er beispielsweise eine geschichtliche Untersuchung zu den jüdischen Personenund Familiennamen in Frankreich (Lévy 1960).

Im Vorwort des 1950 erschienenen ersten Bandes von „La Langue allemande en France“ verwies der Autor nachdrücklich auf das für ihn ausschlaggebende Vorbild der sprachhistorischen Forschungen Ferdinand Brunots (Lévy 1950: VI). Dessen Arbeiten zur Geschichte des Französischen würden durch Lévys eigenen Beitrag zur Wirkung des Deutschen in Frankreich gleichsam erweitert. Ein wirklich in sich geschlossenes Gesamtbild entstünde aber erst dann, so Lévy, wenn eine Geschichte des Englischen, des Spanischen und des Italienischen sowie anderer Sprachen in Frankreich abschließend hinzukäme. Das Endziel wäre somit eine universelle Sprachengeschichte Europas! ${ }^{3}$ Lévys methodisches Vorgehen folgte dem Vorbild Brunots, wenn er die Darstellung an den Epochen der politischen Geschichte Frankreichs ausrichtete. Innerhalb der

3 ,De proche en proche et peu à peu, on arriverait ainsi à une histoire vraiment universelle des langues, d'une part, à une histoire linguistique générale des pays de l'Europe, de l'autre" (Lévy 1950 : VI). 
einzelnen Epochenabschnitte wurde jeweils nach dem gleichen Schema in einem Dreischritt verfahren: erstens ging es um die Präsenz von Deutschen (Studenten, Arbeiter, Söldner etc.) in Frankreich, zweitens um das Verhältnis der Franzosen zur deutschen Sprache, drittens um die Sprachensituation in den deutsch-französischen Grenzgebieten mit ihren Überschneidungen und wechselseitigen Beeinflussungen. Ähnlich wie sein Meister Brunot zog Lévy ein breites Spektrum an Quellentexten heran, um seine Argumentation abzustützen, von amtlichen Verlautbarungen, Lehrbüchern und Bibliotheksverzeichnissen bis hin zu Reiseberichten, Korrespondenzen und Presseartikeln.

Ein Schwerpunkt der Beschreibung liegt für die einzelnen Epochen jeweils bei der Entwicklung in den Grenzgebieten, in denen die Austauschprozesse sich am stärksten verdichteten. Hier hatte Lévy mit seiner Sprachgeschichte von Elsass und Lothringen (Lévy 1929) bereits gut vorgearbeitet. In deren Vorwort wies er auf den besonderen wissenschaftlichen Wert einer Sprachengeschichte des Französischen und des Deutschen in diesem kulturellen Übergangsbereich hin, in dem es zu verdichtetem Kontakt und unterschiedlichsten Austauschbeziehungen kam (Lévy 1929, Bd. 1: 33). Zum anderen betonte Lévy die überragende Bedeutung der politischen Geschichte für die Beziehungen zwischen den beiden Sprachen im Elsass und machte dies an drei Jahreszahlen fest: 1648, 1870, 1918. Diese direkte Rückwirkung von Politik auf die Sprachensituation rechtfertigte gleichsam die Anwendung der sprachenhistorischen Methode Brunots, der sich Lévy zutiefst verpflichtet wusste.

\section{EPOCHEN DER SPRACHENGESCHICHTE IM ELSASS: VON DER VIELHEIT ZUR EINHEIT}

Wenn wir uns auf die Sprachen im Elsass beziehen wollen, so genügt ein Hinweis auf die von Paul Lévy in den Mittelpunkt gestellten Daten 1648, 1870 und 1918, um die maßgebliche Einwirkung der politischen Geschichte auf die linguistische und kulturelle Situation zu demonstrieren. ${ }^{4}$ Mit dem Westfälischen Frieden begann im Jahre 1648 der schrittweise Übergang des gesamten Elsass unter französische Herrschaft, zugleich wurde das territorial uneinheitliche Gebiet erst als Provinz innerhalb des Königreichs Frankreich im 17./18. Jahrhundert politisch und administrativ vereinheitlicht. Dabei wurden lokale Partikularismen und die sprachliche Eigenart der Elsässer zumindest bis zur Revolution (ab 1789) weitgehend respektiert. Generell war die Entwicklung in der Zeit der Zugehörigkeit des Elsass zu Frankreich bis 1870 jedoch von einer langsamen Expansion des Französischen gekennzeichnet, die sich beispielsweise in der Phase der Revolution und des napoleonischen Empire, zwischen 1789 und 1814/15, schubweise beschleunigte (Huck 2015: 73-103). Damit etablierte sich bis zur Mitte des 19. Jahrhunderts die für das Elsass insgesamt kennzeichnende Drei-Sprachen-Ökonomie von Französisch, Deutsch und Elsässisch, die jedem der drei Idiome eine bestimmte Sphäre zuwies: Französisch als Amts- und Elitensprache, elsässische Mundart als gesprochene Sprache der städtischen und der ländlichen Bevölkerung, (Schrift-) Deutsch als

4 Für eine Überblicksdarstellung in deutscher Sprache zur Geschichte des Elsass sei verwiesen auf einen aus einer Mannheimer Ringvorlesung hervorgegangenen Sammelband: Erbe 2002. Daneben beispielhaft auch: Vogler 2003. 
geschriebene Form der Umgangssprache mit einer eindeutigen Prävalenz in (protestantischer) Kirche, in Presse und Literatur (Huck 2015: 105-132). Freilich zeichneten sich ab etwa 1850 eindeutige Verschiebungen innerhalb dieser Ökonomie der drei Sprachen ab, da Französisch zunehmend zu einer gesprochenen Sprache auch der Mittelschicht wurde, die in dieser Hinsicht die Oberschichten nachahmte. In den unteren Schichten herrschten weiterhin die (in der Regel) alemannischen Dialekte des Elsässerditsch vor, so dass soziale Zugehörigkeit auch über die Sprache markiert wurde.

Der Krieg zwischen Frankreich und dem mit den süddeutschen Staaten verbündeten Preußen 1870 verschob die Gewichte in der Sprachenökonomie des Elsass, da mit jedem Herrschaftswechsel auch ein Wechsel der offiziellen Sprache verbunden war. Nach der französischen Niederlage und der Errichtung des deutschen Nationalstaates wurden die Ostgebiete Frankreichs abgetrennt und kamen an das Deutsche Reich, das sie als „Reichsland Elsass-Lothringen“ unter direkte Verwaltung nahm. Nun wurde (Hoch-)Deutsch zur Verwaltungs- und Unterrichtssprache im Elsass, mit der Folge, dass im Lande die bei der Obrigkeit unerwünschten Französischkenntnisse bis zum Jahr 1914 stark zurückgingen (Huck 2015: 151-161). Das Ende des Ersten Weltkrieges 1918 ging dann einher mit einem erneuten Herrschaftswechsel und der Rückkehr an Frankreich, so dass nun Verwaltung und Schule, mit einigen Einschränkungen, wieder komplett zum Französischen wechselten. Besonders die Frage der Unterrichtssprache war unter dem Vorzeichen des verbreiteten Unmutes der um ihre kulturelle Identität besorgten Elsässer (Malaise alsacien) sehr umstritten. 1927 machte Paris hier gewisse Zugeständnisse, im Sinne einer begrenzten Zweisprachigkeit des Schulunterrichtes, wobei das Fach Religion als elsässisches Spezifikum innerhalb der laizistischen Republik ausschließlich in deutscher Sprache gelehrt werden sollte (Huck 2015: 186-190)!

Einer bis dato ungekannten Brutalisierung wurden die Sprachenbeziehungen nach der faktischen Annexion des Elsass durch das nationalsozialistische Deutschland im Sommer 1940 unterworfen. Der Gebrauch des Französischen wurde von den NS-Behörden untersagt. Anders als in anderen Gebieten des Deutschen Reiches, beispielsweise im benachbarten Baden, durfte die Sprache nicht einmal mehr an den Schulen unterrichtet werden. Die NS-Kulturverwaltung übte auch einen gewissen Druck auf die elsässischen Mundarten aus, mit dem Ziel der Durchsetzung einer (hoch-) deutschen Standardsprache ${ }^{5}$ (Kaltz 2016: 239-242). Die Befreiung des Elsass vom Hitlerregime erfolgte unter schweren Kämpfen Ende 1944 und Anfang 1945. Auf die von den Behörden des Dritten Reiches betriebene brutale Germanisierung erfolgte nach der Wiederherstellung der Französischen Republik im Elsass eine Reaktion, die auf eine Verdrängung der (hoch-)deutschen Sprache aus dem öffentlichen Leben zielte. Die elsässischen Dialekte erfuhren dabei zumindest vorübergehend eine gewisse Aufwertung, da sie als „Übergangssprache“ weg vom Deutschen und hin zum Französischen gewertet wurden. So strahlte der Rundfunksender Radio Strasbourg beispielsweise zwischen 1945

5 Zusammenfassend wurde festgestellt, dass die nationalsozialistische Gleichschaltung im Elsass in den Jahren 1940-1942 vor allem vier Zielen verpflichtet war: der Beseitigung der französischen Sprache und Kultur, der Zurückdrängung des Einflusses der (katholischen) Kirche, der Eindeutschung in allen Bereichen und der Durchsetzung der NS-Ideologie (défrancisation, décléricalisation, germanisation, nazification): Grandhomme 2014. 
und 1953 Nachrichtensendung in elsässischer Mundart aus, um jene Hörer im Elsass zu erreichen, die dem französischen Programm (noch) nicht folgen konnten (Huck 2008). Strittig war in der unmittelbaren Nachkriegszeit vor allem die Frage einer Wiederaufnahme des Deutschunterrichts an den Grundschulen, der im Januar 1945 vollständig suspendiert worden war und erst ab 1952 zögerlich wieder eingeführt wurde, doch nur im Rahmen von zwei Wochenstunden und sofern die Eltern dies ausdrücklich für ihre Kinder wünschten (Huck 2006). In paradoxer Weise galt das Deutsche für die elsässischen Schulbehörden in den 1950er und 1960er Jahren wie im übrigen Frankreich als Fremdsprache, deren Erlernen den höheren Schulklassen vorbehalten sei. Im Bereich der Presse galt dagegen eine Verordnung vom 13. September 1945, die vorsah, dass in den elsässischen Zeitungen maximal $75 \%$ der Artikel in deutscher Sprache erscheinen durften, doch mussten Anzeigen und Sportnachrichten mindestens zu $50 \%$, speziell an Jugendliche gerichtete Artikel zu 100 \% auf Französisch verfasst sein (Huck 2015: 236-244).

Diese prinzipielle Zweisprachigkeit elsässischer Presseerzeugnisse blieb bis in die 1970er Jahre erhalten. Solange bewahrte das Deutsche allgemein seine ursprüngliche Position als Schriftsprache. Symptomatisch für das Verschwinden des Deutschen als Idiom der Öffentlichkeit im Elsass war die Volkszählung von 1982, bei der keine Formulare in deutscher Sprache mehr ausgegeben wurden, nachdem beim vorherigen Zensus im Jahr 1975 nur noch sehr wenige dieser Formulare angefordert worden waren (Huck 1985: 111). Das Verschwinden der Standardsprache aus der Sphäre des öffentlichen Lebens sollte durch die Aufwertung der alemannischen Dialekte zur „Regionalsprache Elsässisch" wenigstens teilweise kompensiert werden. ${ }^{6}$ Nach dieser Auffassung spielt das Deutsche im Alltagsleben der elsässischen Bevölkerung keine Rolle (mehr) und kann allenfalls den unmittelbaren geographischen Nachbarn in Deutschland und, in eingeschränktem Maß, in der Schweiz zugeordnet werden. In den 1980er Jahren wurde dann endlich die längst fällige Debatte über die Zukunft der Sprachen des Elsass geführt. Die Vertreter der in der Diskussion besonders involvierten Schulbehörden bezogen dabei durchaus nuancierte Positionen. So äußerte im Jahre 1985 der Leiter des regionalen Schulwesens (Recteur de l'Académie de Strasbourg) Pierre Deyon, dass Elsässisch und Deutsch zentrale Rollen für die regionale Kultur wahrnahmen. Deyon sah das Elsässische als eine „Brückensprache“ hin zum Deutschen. Die enge Verwandtschaft zwischen Dialekt und Dachsprache erleichtere es nicht zuletzt den Schulkindern, die „Sprache des Nachbarn“ (in Deutschland) zu erlernen (Huck 1999: 56). Deyons Nachfolger im Amt, Jean-Paul de Gaudemer, drückte sich 1991 noch grundsätzlicher aus, indem er die Bedeutung des Deutschen betonte, welches zugleich „die verschriftlichte Form der elsässischen Dialekte, die Sprache der nächsten Nachbarländer und eine große europäische Kultursprache" sei (Huck 1999: 58). Damit setzte sich in den 1990er Jahren weitgehend die Konzeption durch, dass Deutsch allenfalls im Sinne des Kulturerbes und der Geschichte eine Regionalsprache im Elsass sei, wohingegen es als Sprache der Nachbarschaftsbeziehungen zu Deutschen und Schweizern eine hervorragende Bedeutung weiterhin beibehalte.

6 Das Elsässische wurde zu einer Schriftsprache mit einheitlichem formalem Regelwerk entwickelt (ORTHAL), vgl. Zeidler/ Crévenat-Werner (2008). 
Beschränkt auf den Status einer „fremden Kultursprache“, spielte Deutsch folglich im Verwaltungsleben der politischen Region Elsass, wie sie bis 2016 bestand, keinerlei Rolle mehr. Diese Situation führte im Alltag zu einem sprachlichen Dualismus von Französisch und Elsässisch, bei dem auch die Mundart nur verlieren konnte. Abgetrennt von der Standardsprache und gebunden an eine ländliche und eher traditionelle Lebensweise, konnte sie der seit den 1960er Jahren beschleunigten gesellschaftlichen Modernisierung nur wenig entgegensetzen. Der Fortschritt sprach gleichsam Französisch und das Elsässische war als Sprache der Vergangenheit und abgelebter Lebensweisen abgewertet. Die Hinwendung zumal jüngerer Frauen zu dieser sprachlich konnotierten Modernität führte zwischen 1960 und 1980 zu einem Bruch in der Sprachvermittlung, der letztlich irreparabel war. Französisch galt in dieser Zeit als Sprache der Moderne, der Emanzipation und des sozialen Aufstieges für die Kinder. Die Mundart im Elsass war somit in diesem Zeitraum nicht genuin das „Opfer“ einer an nationalen Prioritäten ausgerichteten Sprachpolitik, sondern sie geriet unter den Druck sozialer Umbrüche, die letztlich aus dem Verschwinden der traditionellen Agrargesellschaft herrührten (Huck 2015: 319-368). Damit kann für das Elsass in der zweiten Hälfte des 20. Jahrhunderts eine Normalisierung der Sprachensituation, im Vergleich zu anderen französischen Regionen, festgestellt werden. An die Stelle der Vielheit in einer seit dem 17. Jahrhundert stabilisierten Drei-Sprachen-Ökonomie ist eine weitgehende Vereinheitlichung getreten, in der das Elsässische als „Regionalsprache“ einen geschützten Status genießt, der seinen Bedeutungsverlust kaum verdeckt und in der sich die einst im Elsass präsente Schriftsprache Deutsch seit den 1980er Jahren im europäischen Kontext einer gewissen Wertschätzung als die „Sprache des Nachbarn“ erfreut, während in allen Bereichen das Französische den Alltag beherrscht und prägt.

\section{DIE REPUBLIK KENNT KEINE MINDERHEITEN: DAS PROBLEM DER „SPRACHEN FRANKREICHS“}

Im Präsidentschaftswahlkampf 1981 hat der sozialistische Kandidat und spätere Wahlgewinner François Mitterrand (1916-1996) mit der Forderung Aufsehen erregt, dass man die Schulen und den Unterricht für die Sprachen Frankreichs weit öffnen müsse: „Ouvrir grandes les portes de l'école aux langues de France“. Welches aber sind die Sprachen Frankreichs? Dieser Frage haben sich seither zahlreiche Kolloquien und Podiumsveranstaltungen gewidmet (u. a. Klein 2013). Diese Veranstaltungen umkreisen oft das Problem, dass die Französische Republik zwar die 1992 vom Europarat angenommene Europäische Charta der Regional- und Minderheitensprachen paraphiert, sie aber dann nicht ratifiziert hat. In der Tat fiel die Verabschiedung der Charta zeitlich mit der Revision der Verfassung der Fünften Republik zusammen (1992), bei der erstmals in der Geschichte französischer Verfassungen eine Feststellung hinsichtlich der Sprache getroffen wurde (Artikel 2: La langue de la République est le français). Dieses Prinzip schien wenig Spielraum für die Entfaltung anderer Sprachen in der Republik zu lassen, doch wurde es bei einer weiteren Verfassungsrevision im Jahr 2008 dahingehend relativiert, dass nun auch die Regionalsprachen als zum Kulturerbe Frankreichs gehörig im Verfassungstext implementiert wurden (Artikel 75-1: Les langues 
régionales appartiennent au patrimoine de la France) (Weiss 2017: 275-280). Der Begriff der Minderheitensprache hingegen ist mit dem in der französischen Verfassungstradition seit 1789/1791 betonten Gleichheitsprinzip unvereinbar und darin liegt auch zu einem guten Teil das Problem mit der Europarats-Charta von 1992 begründet.

Mit dem 2008 in die Verfassung eingefügten Begriff der Regionalsprache ist freilich das Deutsche nicht gemeint, schon weil die Elsässer und die Lothringer (im Département Moselle bei Metz) dies nicht wollten. Es wurde darauf verweisen, dass dem Deutschen in diesen Gebieten stets eine wichtige Eigenschaft fehlte, nämlich gesprochene Sprache zu sein. Es war allenfalls „Schriftsprache“ und teilte sich diese Funktion seit dem 17. Jahrhundert mit dem Französischen. Hinzuweisen ist auch auf die nach 1871 von den Elsässern selbst betriebene Aufwertung des Elsässerditsch zur kulturellen Abgrenzung von den „Altdeutschen“ aus den übrigen Bundesstaaten des Deutschen Reiches. Eine wichtige Rolle spielte dabei das Mundarttheater, das ab 1898 einen bemerkenswerten Aufschwung erlebte, ausgelöst durch den großen Erfolg der Uraufführung des lange Zeit äußerst populären Stückes D'r Herr Maire von Gustave Stoskopf (1869-1944), einem der Mitbegründer des den Stücken im elsässischen Dialekt gewidmeten Théâtre alsacien in Straßburg (Huck 2005). Dieses Theater war ein Ort der regionalen Selbstvergewisserung und der Abgrenzung von den „Schwaben“ (Schwowe) genannten Deutschen von jenseits des Rheines. ${ }^{7}$ Eine wichtige Rolle in diesen Strategien der Distanzierung gegenüber den durch Einwanderung massiv präsenten „Altdeutschen“ übernahm nach 1871 der frankophile katholische Klerus, hatte Elsass-Lothringen doch den höchsten Katholikenanteil unter allen „Ländern“ des Deutschen Reiches (etwa $75 \%$ ).

Die Betonung einer eigenen elsässischen Sprache und Kultur, die sich weiterhin über die Nähe zu Frankreich definierte, war gegen die Dominanz des preußisch-norddeutschen Kulturprotestantismus gerichtet, der eines der kulturellen Fundamente des Bismarck-Reiches bildete. Deutlich wurde diese auf Eigenständigkeit bedachte Haltung des Klerus beispielhaft an der Biographie des Abbé Émile Wetterlé (1861-1931), eines betont politischen Geistlichen, von 1898 bis 1915 Abgeordneter im Deutschen Reichstag und 1919 bis 1924 in der französischen Nationalversammlung (Baechler 1986). Wetterlé hatte den mentalen Bruch mit der deutschen Herrschaft im Elsass bereits vor 1914 vollzogen und entschied sich bei Beginn des Ersten Weltkrieges für Frankreich, was in logischer Folge zum Verlust seines Reichstagsmandates in Berlin führte. Die frankreichfreundliche Position des politisierenden Abbé Wetterlé ähnelte in vielen Punkten der Preußenkritik führender elsässischer Künstler und Intellektueller des Kreises um Charles Spindler (1865-1938), der 1898 die Zeitschrift Revue alsacienne illustrée gründete, die sich der Pflege von Brauchtum und Kultur im Elsass, ebenfalls in Abgrenzung zu den preußischdeutschen Vereinnahmungstendenzen, widmete (Orzechowski 1998). Alle diese mit großem Engagement verbundenen Bestrebungen verweisen auf die „Fremdheit“ des Standarddeutschen im Elsass, ähnlich wie in der heutigen Schweiz, die es für die Funktion einer Regionalsprache ungeeignet erscheinen ließ. Letztlich bedurfte es dafür kaum noch der zutiefst belastenden Erfahrungen der Kriegsjahre 1940-1945, als die NS-Behörden

7 Zur Blüte der elsässischen Dialektliteratur um 1900 allgemein: Wackenheim 1997; Finck/Staiber (2004: 27-51). 
das Französische mit einem Verbot belegten und selbst die elsässischen und badischen Mundarten am Oberrhein bekämpften, da der damit verbundene lokale Eigensinn den Ansprüchen einer totalitären Diktatur widerstrebte. Die Brandmarkung des (Hoch-)Deutschen als „Sprache der Nazis“ nach 1945 hat dessen Ausscheiden aus der Sprachenökonomie des Elsass wesentlich gefördert.

\section{ZUM SCHLUSS}

Auch wenn sich der erwähnte Begriff der gefühlten Fremdheit wissenschaftlicher Kategorisierung entzieht, beschreibt er doch den Status der deutschen Sprache in Frankreich, zumal in den von Paul Lévy besonders untersuchten Grenzgebieten. Als junger Lehrer im Reichsland Elsass-Lothringen plädierte dieser 1913 in einer engagierten Schrift, die sich vor allem an die Kollegen in den Schulen des Reichslandes richtete, für die Einbeziehung der elsässischen Dialekte in den Deutschunterricht (Lévy 1913). Er wandte sich damit gegen die von der elsass-lothringischen Schuldirektorenkonferenz 1873 erlassenen Grundsätze, die vom Schüler ein „,mustergültiges Schriftdeutsch“ forderten. Dagegen wandte Lévy ein, dass Elsässisch ,in ganz hervorragendem Maße in der Schule anwendbar“ sei (Lévy 1913: 11). Die Mundart bezeichnete er als ein „Mittel für Wahrhaftigkeit und gegen Phrasentum“, denn sie fördere genaues Bobachten und Denken, schließlich bringe sie „Schüler und Lehrer, Schule und Haus, hoch und niedrig sich gegenseitig näher" (ebenda: 32). Der entscheidende Vorteil des Dialekts gegenüber der Hochsprache sei

die Tatsache, dass er die Natürlichkeit und die Korrektheit des Denkens fördere: „Überall, wo sich im Aufsatz Prunk und Wichtigtuerei und hohles Wortgeklingel ohne richtige Anschauung breitmachen wollen, vermögen sie am besten wohl durch den Hinweis auf die Sprödigkeit und Nüchternheit der Mundart, der trotzdem nie die Wärme und Treffsicherheit fehlen, berichtigt zu werden" (ebenda: 23). Während die im Elsass auch nach vierzig Jahren der Zugehörigkeit zum deutschen Nationalstaat als fremd empfundene deutsche Hochsprache die verderbliche Neigung zu realitätsfernem und abstraktem Gerede befördere, habe die Mundart den Vorteil der Wirklichkeitsnähe und des Bezuges zu den Dingen des Alltages. Es ist Lévy sicherlich Recht zu geben, wenn er in den gängigen deutschen Diskursen seiner Zeit einen allzu geringen Gehalt an Wahrheit und Wahrhaftigkeit ausmachte und dies auch auf den Abstand zwischen dem Schriftdeutschen und der gesprochenen Sprache zurückführte. Sein Plädoyer von 1913 für einen Ausgleich zwischen Hochsprache und Mundart verweist auf ein bis heute nicht zufriedenstellend gelöstes Problem in der durch die politischen Verwerfungen im 19./20. Jahrhundert immer wieder umgewälzten Sprachenökonomie des Elsass, als markantem Teilkomplex des großen Themas der „,eutschen Sprache in Frankreich“.

\section{Literatur}

BAECHLER, Christian (1986) „L'abbé Wetterlé, un prêtre patriote et libéral.“ Archives de l'Église d'Alsace, 45, 243-286.

BRUNOT, Ferdinand (1933) Histoire de la langue française des origines à 1900, 13 Bde. Paris: Colin [Nachdruck: Paris: Colin, 1966-1979]. 
HAFNER, Jochen (2006) Ferdinand Brunot und die nationalphilologische Tradition der Sprachgeschichtsschreibung in Frankreich. Tübingen: Narr.

ERBE, Michael (2002) Das Elsass. Historische Landschaft im Wandel der Zeiten. Stuttgart: Kohlhammer.

FINCK, Adrien/Maryse STAIBER (2004) Histoire de la littérature européenne d'Alsace. Vingtième Siècle. Strasbourg: Presses universitaires.

GRANDHOMME, Jean-Noël (2014) „La ,mise au pas“ (Gleichschaltung) de l’AlsaceMoselle en 1940-1942: défrancisation, décléricalisation, germanisation, nazification." Revue d'Allemagne, 46, 443-465.

HUCK, Dominique (1985) „Elsässerdeutsch ohne Deutsch. Zur Lage der Mundart und der deutschen Standardsprache im Elsaß.“ In: A. Ritter (Hrsg.), Kolloquium zur Sprache und Sprachpflege der deutschen Bevölkerungsgruppen im Ausland. Flensburg: Institut für Regionale Forschung und Information im Deutschen Grenzverein, 107-130.

HUCK, Dominique (1999) „Quelle ,langue régionale“ en Alsace ?“ In: L. Dabène (Hrsg.), Les langues régionales. Enjeux sociolinguistiques et didactiques. LIDIL. Revue de Linguistique et de Didactique des Langues Université Stendhal de Grenoble). 20, 43-60.

HUCK, Dominique (2005) Le 'théâtre alsacien de Strasbourg' et la production dramaturgique de ses fondateurs (1898-1914). Bern: Peter Lang.

HUCK, Dominique (2006) „L'enseignement de l'allemand à l'école primaire en Alsace entre 1945 et 1985.“ Revue d'Alsace, 132, 337-406.

HUCK, Dominique (2008) „Les politiques linguistiques et les dialectes en Alsace depuis 1945.“ In : E. Béhague/D. Goeldel (Hrsg.), Une germanistique sans rivages. Mélanges en l'honneur de Frédéric Hartweg. Strasbourg: Presses universitaires, 56-64.

HUCK, Dominique (2015) Une histoire des langues d'Alsace. Strasbourg: La Nuée Bleue.

KALTZ, Barbara (2013) Paul Lévy, Die deutsche Sprache in Frankreich. 1: Von den Anfängen bis 1830. Aus dem Französischen übersetzt und bearbeitet von Barbara Kaltz. Wiesbaden: Harrassowitz.

KALTZ, Barbara (2016) Paul Lévy, Die deutsche Sprache in Frankreich. 2: Von 1830 bis 1944. Aus dem Französischen übersetzt und bearbeitet von Barbara Kaltz. Wiesbaden: Harassowitz.

KLEIN, Pierre (2013) Les langues de France et la ratification de la charte européenne de langues régionales ou minoritaires. Colloque de Strasbourg. Strasbourg: Initiative citoyenne alsacienne pour plus de démocratie.

LÉVY, Paul (1908) „Lettres nouvelles de Nietzsche.“ La revue. Ancienne revue des revues. 76, 185-205.

LÉVY, Paul (1911) Geschichte des Begriffes Volkslied. Berlin: Mayer \& Müller.

LÉVY, Paul (1913) Die Verwertung der Mundarten im Deutschunterrichte höherer Lehranstalten unter besonderer Berücksichtigung des Elsässischen. (= Zeitschrift für den deutschen Unterricht. 8. Ergänzungsheft). Leipzig/Berlin: Teubner.

LÉVY, Paul (1923) „Les dialectes germaniques en Lorraine, essai de bibliographie critique." Annuaire de la Société d'histoire et d'archéologie de Lorraine. 36, 261-276. 
LÉVY, Paul (1929) Histoire linguistique d'Alsace et de Lorraine. 2 Bde. Paris : Les Belles Lettres (Publications de la Faculté de Lettres de l'Université de Strasbourg 47/48) [Nachdruck Houilles : Manucius 2004].

LÉVY, Paul (1938) „Les romantiques français et la langue allemande“. Revue germanique, 29, 225-252.

LÉVY, Paul (1950/52) La langue allemande en France: pénétration et diffusion des origines à nos jours. 2 Bde. Lyon/Paris: IAC.

LÉVY Paul (1960) Les noms des israélites en France. Histoire et dictionnaire. Paris: Presses universitaires de France.

ORZECHOWSKI, Simone (1998). „La 'Revue alsacienne illustrée' (1898-1914). L'art, paravent de la lutte contre la germanisation.“ In: M. Grunewald (Hrsg.), Le problème de l'Alsace-Lorraine vu par les périodiques (1871-1914)/ Die elsasslothringische Frage im Spiegel der Zeitschriften (1871-1914). Bern: Peter Lang, 413-431.

VOGLER, Bernard (2003) Nouvelle Histoire de l'Alsace. Une région au cœur de l'Europe. Toulouse: Éditions Privat.

WACKENHEIM, Auguste (1997) La Littérature dialectale alsacienne. Une anthologie illustrée, Tome 3: La période allemande (1870-1918). Paris: Prat.

WEISS, Saskia (2017) Europäischer Minderheitenschutz am nationalen Beispiel der Regionalsprachen in Frankreich. Frankfurt a. Main: Peter Lang.

ZEIDLER, Edgar/Danielle CRÉVENAT-WERNER (2008) Orthographie alsacienne: bien écrire l'alsacien de Wissembourg à Ferrette. Colmar: Bentzinger.

\section{Zusammenfassung \\ DIE DEUTSCHE SPRACHE IN FRANKREICH, MIT BESONDERER BERÜCKSICHTIGUNG DES ELSASS - EIN HISTORISCHES THEMA UND SEINE AKTUELLEN ABLEITUNGEN}

Um das Gesamtbild von den Minderheitensprachen abzurunden, soll auch ein Blick auf die ganz andere Situation im westlichen Europa gerichtet werden. In Frankreich wird seit langem eine historische Soziolinguistik der Sprachen betrieben. Für diese sind die Arbeiten Paul Lévys (1887-1962) kennzeichnend, der sich intensiv mit der Geschichte der deutschen Sprache in Frankreich befasst hatte. Ein interessanter Beispielfall ist dabei das Elsass, aus dem der Linguist und Historiker Lévy stammte. In dem Aufsatz geht es darum, die Besonderheiten der Sprachenentwicklung im französischen Elsass summarisch aufzuzeigen, die als Vergleichsmaßstab für Sprachenpolitik im Flächenstaat dienen kann.

Schlüsselwörter: Paul Lévy (1887-1962), historische Soziolinguistik (Frankreich), Elsass (17.-21. Jahrhundert), französische Sprachenpolitik, Minderheitensprachen westliches Mitteleuropa 


\section{Abstract \\ THE GERMAN LANGUAGE IN FRANCE, WITH A PARTICULAR FOCUS ON ALSACE - ITS HISTORY AND PRESENT IMPLICATIONS}

This paper seeks to contribute to the study of minority languages by shedding light on their position and status in Western Europe. In France, there is a long-established tradition of historical sociolinguistic research, notably represented by the historian and linguist Paul Lévy (1887-1962), who has made important research on the history of the German language in France. Originally from Alsace, Lévy published a number of works on the linguistic specificities of this part of the French Republic. The paper outlines the most important points of the sociolinguistic evolution of Alsatian, which may be a point of reference for further reflection on language policies in multilingual nation states.

Keywords: Paul Lévy (1887-1962), historical sociolinguistics (France), Alsace (17 $7^{\text {th }}$ $21^{\text {st }}$ centuries), language policy in France, minority languages in Western Europe

\section{Povzetek}

NEMŠKI JEZIK V FRANCIJI, S POSEBNIM POUDARKOM NA ALZACIJI ZGODOVINSKA TEMATIKA IN NJENE AKTUALNE IZPELJAVE

Prispevek ima namen dopolniti celostno podobo manjšinskih jezikov z osvetlitvijo jezikovnomanjšinske situacije $\mathrm{v}$ zahodni Evropi. V Franciji so historične sociolingvistične razprave že dolgo aktualne. Zaznamovala so jih dela Paula Lévyja (1887-1962), v katerih se je intenzivno ukvarjal z zgodovino nemškega jezika v Franciji. Pri tem je še posebej zanimiva Alzacija, od koder jezikoslovec in zgodovinar Lévy tudi izvira. Cilj prispevka je prikazati sociolingvistične posebnosti jezikovnega razvoja $\mathrm{v}$ tej francoski regiji, za jezikovno politiko v državi pa razvoj v Alzaciji lahko služi s primerjalnega vidika.

Ključne besede: Paul Lévy (1887-1962), historična sociolingvistika (Francija), Alzacija (17.-21. stoletje), francoska jezikovna politika, manjšinski jeziki zahodne Srednje Evrope 